\title{
An attitude measurement method to compensate the error of algorithm precision based on three-axis gyroscope

\author{
Yixiang Shi ${ }^{1,2,3, a}$, Ke Chen ${ }^{1,2, b,{ }^{*}}$ and Deyi Chen ${ }^{1,2,3, c}$
}

${ }^{1}$ Key Laboratory of Optical Engineering, Chinese Academy of Sciences, Chengdu 610209, China

2Institute of Optics and Electronics, Chinese Academy of Sciences, Chengdu 610209, China

3 University of Chinese Academy of Sciences, Beijing 100049, China

ashiki_yx@126.com, bckxjpl@yahoo.com.cn, c1217379985@qq.com

${ }^{*}$ Corresponding author

Keywords: Attitude measurement, Angular-rate integral, Error compensation

Abstract. In order to compensate the error of angular-rate integral and improve the precision when it comes to measure the attitude of carrier, a method based on 3-axis gyroscope is proposed. The movement of carrier is viewed as time series based on sampling interval, and two adjacent measurements are relative. The difference between two adjacent measurements will reveal the trend of angular rate, which can be used to adjust the angular rate and correct the measurements of gyroscope to compensate the error of algorithm. Experiment results show that the proposed method can get a more accurate attitude information of carrier through compensation and the simulation in different sampling frequencies are consistent.

\section{一种基于三轴陀螺仪的姿态解算误差补偿方法 \\ 史一翔 $1,2,3, \mathrm{a}$ ，陈科 $1,2, \mathrm{~b},{ }^{*}$ ，陈德毅 $1,2,3, \mathrm{c}$ \\ 1中国科学院光束控制重点实验室, 成都, 四川, 中国, 610209 \\ 2中国科学院光电技术研究所, 成都, 四川, 中国, 610209 \\ 3中国科学院大学, 北京, 中国, 100049 \\ ashiki_yx@126.com, bckxjpl@yahoo.com.cn, ${ }^{c 1217379985 @ q q . c o m ~}$ \\ *通讯作者}

关键词: 姿态解算; 角速率积分; 误差补偿

中文摘要. 为了对姿态测量中基于陀螺仪角速率积分的角增量算法进行误差补偿修正, 提高 载体姿态的测量精度, 提出了一种基于三轴陀螺仪的姿态解算误差补偿方法。将载体的运动 看成是采样间隔下的时间序列, 相邻两测量值间具有相关性, 二者的差值可用于推断下一时 刻角速率的变化趋势, 以此来对当前时刻角速率进行调整, 修正当前陀螺仪测量值, 实现姿 态解算误差补偿。实验结果表明, 经过此方法修正补偿可得到更为精确的载体姿态信息, 不 同采样频率下的实验结果表明该方法一致性较好。

\section{1. 引言}

惯性导航技术是基于陀螺仪和加速度计来实现定位定向的一门技术，姿态测量技术作为 其中的关键部分, 决定着导航系统的测量精度 ${ }^{[1]}$ 。其中惯性器件陀螺仪用于对角速率进行测 
量, 它是一个间接测量器件, 其测量的对象角速率是角度关于时间的导数, 积分后得到相对 角度, 由相对角度所维持的姿态矩阵可用于对物体的姿态进行表示。

由积分原理可知，当积分时间越小时，由陀螺仪输出角速率计算出的角度与真实旋转的 角度值越接近, 但受限于器件的性能和系统的处理能力, 数据的采样频率并不能无限提高, 即采样间隔时间不可能无限小。这也就使得测量出的姿态信息与真实的姿态信息之间存在算 法原理性上的误差, 如何对这一误差进行补偿, 使计算出的姿态信息与真实姿态信息尽可能 接近, 是本文的研究重点。

\section{2. 载体姿态测量坐标系变换}

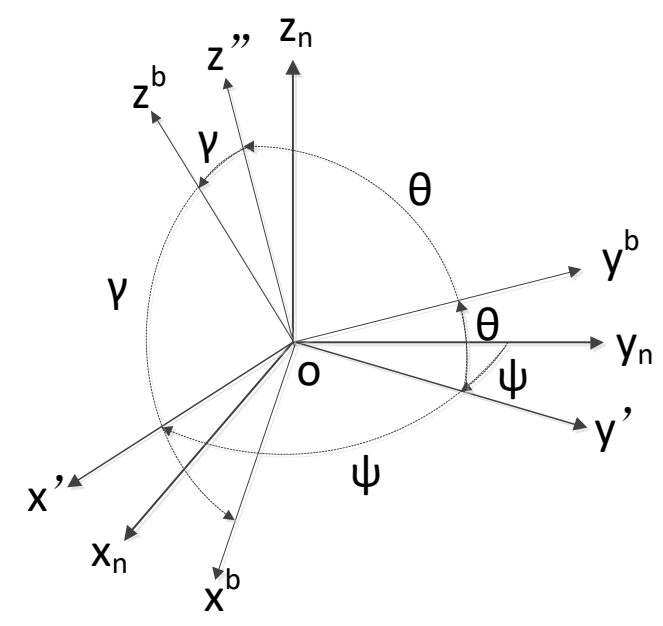

图1 载体姿态旋转示意图

载体坐标系与导航坐标系间的变换矩阵称为姿态矩阵 $C_{b}^{n}$, 如图1所示, 其中 $\psi$ 、 $\theta$ 和 $\gamma$ 分 别为载体姿态中的偏航角 Yaw、俯仰角 Pitch 和滚转角 Roll, 在大多数情况下, 载体的空间姿 态可由当地地理坐标系, 先后沿着自身的偏航轴、俯仰轴、横滚轴旋转相应角度之后得到 ${ }^{[2]}$, 即

$$
O X_{n} Y_{n} Z_{n} \stackrel{\text { 绕偏航轴旋转 }-\psi}{\longrightarrow} O X^{\prime} Y^{\prime} Z^{\prime} \stackrel{\text { 绕俯仰轴旋转 } \theta}{\longrightarrow} O X^{\prime \prime} Y^{\prime \prime} Z^{\prime \prime} \stackrel{\text { 绕横滚轴旋转 } \gamma}{\longrightarrow} O X_{b} Y_{b} Z_{b}
$$

其中旋转矩阵的关系如下 ${ }^{[3]}$

$$
\begin{aligned}
R_{z}(-\psi) & =\left[\begin{array}{ccc}
\cos \psi & -\sin \psi & 0 \\
\sin \psi & \cos \psi & 0 \\
0 & 0 & 1
\end{array}\right] \\
R_{x}(\theta) & =\left[\begin{array}{ccc}
1 & 0 & 0 \\
0 & \cos \theta & \sin \theta \\
0 & -\sin \theta & \cos \theta
\end{array}\right] \\
R_{y}(\gamma) & =\left[\begin{array}{ccc}
\cos \gamma & 0 & -\sin \gamma \\
0 & 1 & 0 \\
\sin \gamma & 0 & \cos \gamma
\end{array}\right]
\end{aligned}
$$

根据相关规则, 对应的姿态矩阵为 


$$
\begin{aligned}
& C_{n}^{b}=\left[C_{b}^{n}\right]^{\mathrm{T}}=\left[R_{z}(-\psi) R_{x}(\theta) R_{y}(\gamma)\right]^{\mathrm{T}}= \\
& {\left[\begin{array}{ccc}
\cos \psi \cos \gamma-\sin \psi \sin \theta \sin \gamma & \sin \psi \cos \gamma+\cos \psi \sin \theta \sin \gamma & -\cos \theta \sin \gamma \\
-\sin \psi \cos \theta & \cos \psi \cos \theta & \sin \theta \\
\cos \psi \sin \gamma+\sin \psi \sin \theta \cos \gamma & \sin \psi \sin \gamma-\cos \psi \sin \theta \cos \gamma & \cos \theta \cos \gamma
\end{array}\right]}
\end{aligned}
$$

运动状态下的姿态由当前时刻姿态矩阵与当期角增量矩阵相乘表示，即

$$
C_{n}^{b}(t+1)=C_{n}^{b^{\prime}}(t) C_{b^{\prime}}^{b}(\Delta t)
$$

由当前姿态矩阵计算当前姿态角

$$
\left\{\begin{array}{l}
\text { Yaw }=\psi=\arctan \frac{-C_{n}^{b}(2,1)}{C_{n}^{b}(2,2)} \\
\text { Pitch }=\theta=\arcsin C_{n}^{b}(2,3) \\
\text { Roll }=\gamma=\arctan \frac{-C_{n}^{b}(1,3)}{C_{n}^{b}(3,3)}
\end{array}\right.
$$

\section{3. 算法验证模型}

物体姿态由俯仰角 Pitch、横滚角 Roll、方位角Yaw 构成, 在选定参考系的基础上, 姿态 解算的目的是确定载体坐标系与参考坐标系的数学关联, 通常采用姿态矩阵表示 ${ }^{[4]}$ 。为求得 姿态矩阵, 需要选取合理的姿态解算方法。传统的姿态解算算法有欧拉角法、方向余弦法以 及四元数法 ${ }^{[5]}$ 。

其中，载体在全姿态运动条件下，欧拉角微分方程在解算过程中易产生奇异点问题，且 三角运算较多，计算复杂，因此欧拉角法仅适合在近似水平姿态条件下的姿态解算，在工程 应用中具有一定的局限性。方向余弦法尽管解算过程中不会出现奇异点问题, 可实现全姿态 条件下的数学解算, 但由于涉及的参数较多, 计算复杂, 对系统实时性影响较大, 不利于工 程应用。相比以上算法, 四元数法不仅适用于全姿态条件下的姿态解算, 而且计算量小, 实 时性较好, 可广泛应用于工程实践中。本文选用毕卡法计算角增量, 采用四元数法对姿态进 行表示，检验算法的算法误差 ${ }^{[6]}$ 。

为了对算法精度进行验证, 因此不对器件误差和初始标定等因素进行考虑, 即将标定误 差、安装误差和陀螺仪器件误差视为 0 , 仅从姿态解法上讨论积分误差。

实验原理图如下:

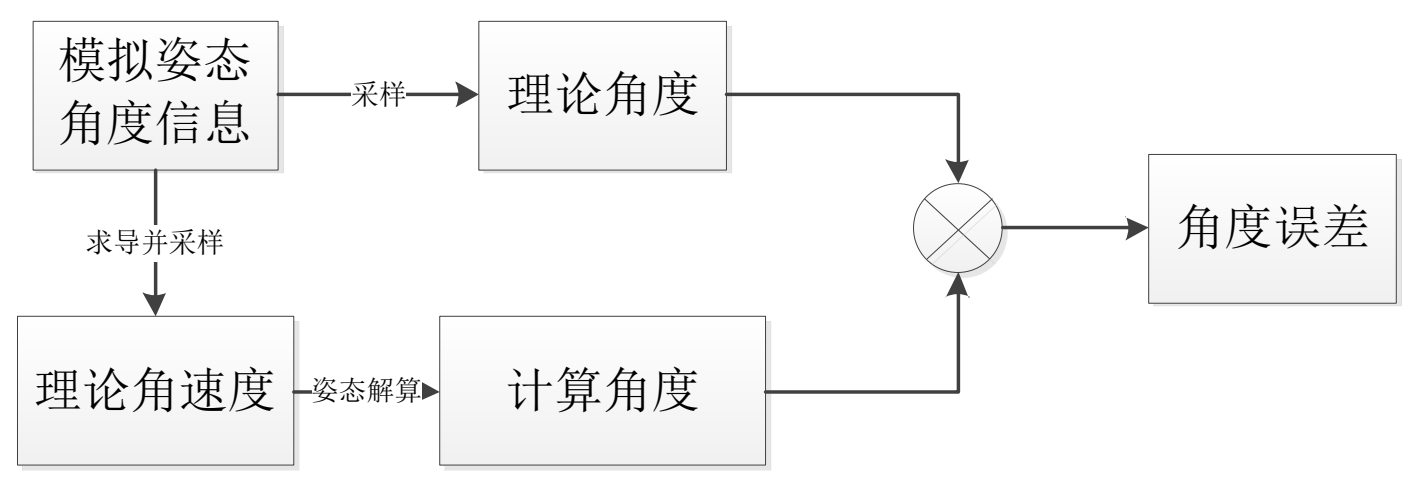

图2 算法验证模型示意图

被测物体姿态由俯仰角、横滚角、方位角构成，模拟三个姿态角度信息，均使用正弦函 数作为输入角度理论值, 其形式为 
上述函数对时间求导，得到理论角速率信息为

$$
\left\{\begin{array}{l}
t \text { Pitch }=A \sin \omega t \\
t R o l l=A \sin \omega t \\
t Y a w=A \sin \omega t
\end{array}\right.
$$

$$
\left\{\begin{array}{l}
d t \text { Pitch }=A \omega \cos \omega t \\
d t R o l l=A \omega \cos \omega t \\
d t Y a w=A \omega \cos \omega t
\end{array}\right.
$$

因为仅对算法积分误差进行讨论，不考虑其他因素，因此将理论角速率等同于实际角速 率

$$
\left\{\begin{array}{l}
d \text { Pitch }=d t \text { Pitch } \\
d \text { Roll }=d t \text { Roll } \\
d \text { Yaw }=d t \text { Yaw }
\end{array}\right.
$$

对角速率信息进行积分, 求解被测物体姿态角度, 再由计算出的姿态角减去同一采样时 刻的理论姿态角, 其差值即为算法的原理性误差。

\section{4. 误差分析与改进方案}

在实际应用中, 由于数据采样频率受到诸多限制, 使得采样间隔不能无限缩小, 因此由 积分累计计算出的姿态角度客观上存在原理性误差。

以单轴陀螺仪为例对角增量误差进行分析, 如图所示, 横轴表示时间, 纵轴表示角度。

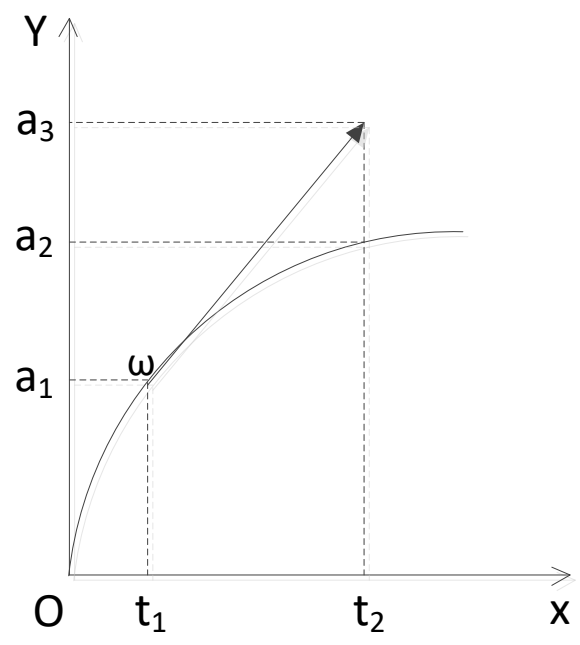

图3 积分误差示意图

相关参数如下:

$$
\left\{\begin{array}{l}
\Delta t=t_{2}-t_{1} \\
a_{3}=a_{1}+\omega * \Delta t \\
\text { tAngle }=a_{2}-a_{1} \\
\text { Angle }=a_{3}-a_{1} \\
\Delta \text { Angle }=\text { Angle-tAngle }=a_{3}-a_{2}
\end{array}\right.
$$

其中 $\Delta t$ 表示采样时间间隔, tAngle 表示真实角增量, Angle 为计算角增量, $\Delta A n g l e$ 即为 原理性误差, 即在本文中, 需要进行修正和补偿的部分。 
为了对 $\Delta A n g l e$ 这一误差进行补偿, 需要采用相应方法对测算角速度进行修正, 调整相对 应计算得出角度的大小，使得其与真实角度的差值尽可能小;

由于姿态角度的变化是一个连续的过程，其相对于时间的导数，即角速率也具有连续性。 因此采样得到的时间序列中相邻两角速率之间存在强相关性, 二者的差值可用于推断下一时 刻角速率的变化趋势，以此来对当前时刻角速率进行调整。

对角速率测量进行修正, 等效角速率 gyro $(t)$ 由理论角速率 tgyro $(t)$ 和调整因子 $\Delta$ tgyro 构 成，如下所示:

$$
\left\{\begin{array}{l}
\text { gyro }(t)=\alpha * \operatorname{tgyro}(t)+\beta * \Delta \text { tgyro } \\
\Delta \text { tgyro }=\operatorname{tgyro}(t)-\operatorname{tgyro}(t-1)
\end{array}\right.
$$

\section{5. 试验结果与分析}

本文基于毕卡法对四元数姿态信息进行解算，相关参数设置如下:

$$
\left\{\begin{array}{l}
A=40^{\circ} \\
\omega=0.039 \mathrm{~Hz} \\
\Delta \mathrm{t}=0.005 \text { sor } 0.001 \mathrm{~s} \\
\mathrm{~T}=300 \mathrm{~s}
\end{array}\right.
$$

其中 $A$ 和 $\omega$ 为输入正弦函数的振幅和频率， $\mathrm{T}$ 为采样时间， $\Delta \mathrm{t}$ 为采样间隔时间。对算法 进行仿真验证, 结果如下图所示。
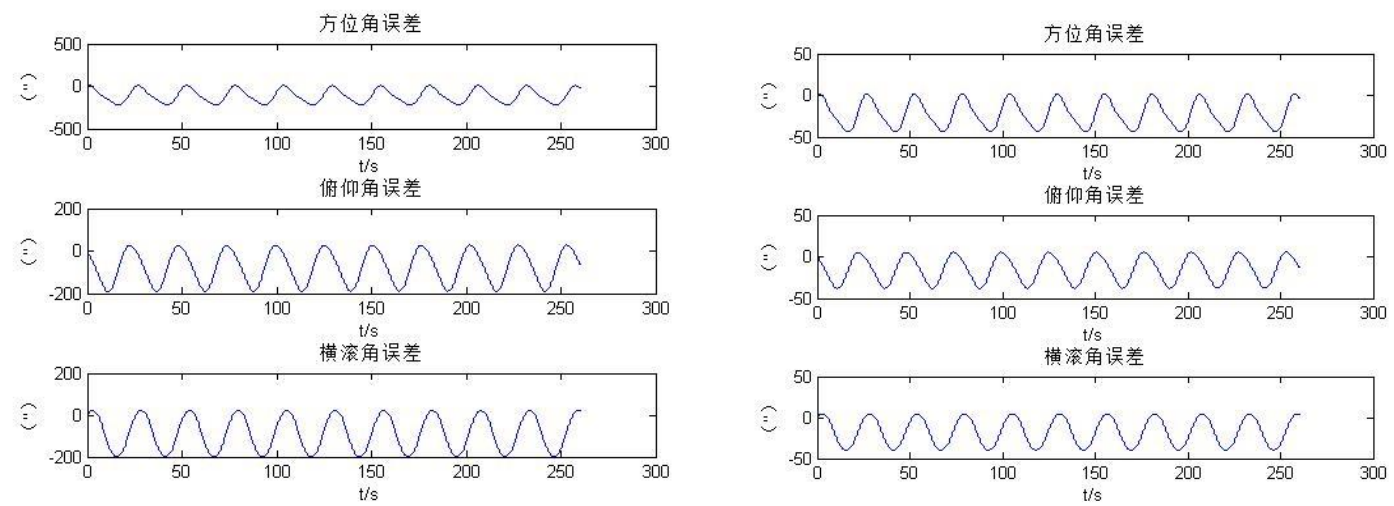

图4 采样时间为 0.005 秒和 0.001 秒时实验结果示意图

当采样频率为 $200 \mathrm{~Hz}$ 时, 算法误差在 200 角秒内, 提升采样频率到 $1000 \mathrm{~Hz}$, 算法误差也接 近40角秒。可知, 提升采样频率可使计算角度与真实角度更加接近, 但由于器件性能及系统 处理能力的限制, 使得采样频率只能做有限提升。因此, 在采样频率限制下, 对计算出的相 应姿态角度误差进行补偿, 减少其与真实角度值的差值, 对高精度姿态测量来说十分重要。

在对角速率值进行修正后, 在同样条件下进行实验, 其姿态相对角度误差如下图所示: 

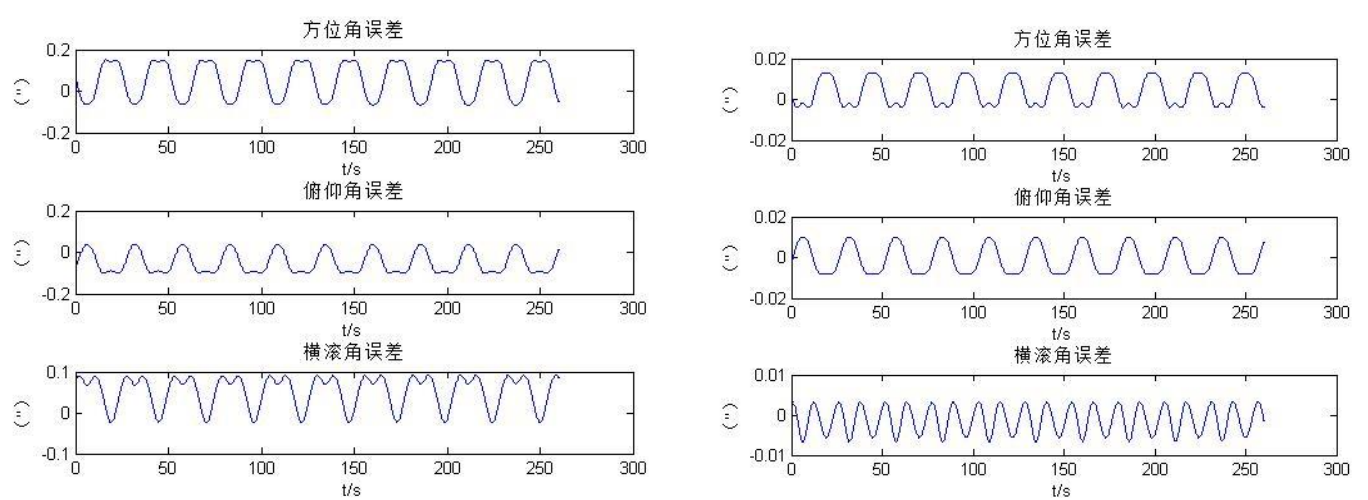

图5 修正后采样时间为 0.005 秒和 0.001 秒时实验结果示意图

经过测算比较, 当 $\alpha=1, \beta=-0.5$ 时, 对算法误差修正效果最好, 采样频率 $200 \mathrm{~Hz}$ 时, 算 法误差在 0.2 角秒内, 采样频率 $1000 \mathrm{~Hz}$ 时, 算法误差在 0.02 角秒内, 与未进行修正相比, 算法 精度提升较大。

\section{6. 结论}

惯性器件受限于器件性能, 采样频率无法无限提升, 使得角速率积分存在算法原理性上 误差, 因此对于高精度姿态测量来说, 对测量出的角速率信息进行调整, 使所计算出的角度 值与理论角度值误差缩小, 具有重要意义。在本文中, 针对基于三轴陀螺仪的毕卡四元数姿 态测量算法提出了一种误差补偿方法, 经仿真实验验证, 可以明显改善算法精度。

\section{References}

[1] Quan W, Fang J C, Xu F, et al, Hybrid Simulation System Study of SINS/CNS Integrated Navigation. IEEE Aerospace and Electronic Systems Magazine, 2008, 23(2): 17-24

[2] Paul G.. Savage Strapdown Inertial Navigation System Algorithm Design, Part 2: Velocity and Position Algorithms, AIAA Journal of Guidance, Control and Dynamics. 1998,21(2): 208-211

[3] Hongwei Qin, Research on SINS/STAR Integrated Navigation Technology[D], Harbin Institute of Technology, China, 2013

[4] Zhihua Wu, Research on Attitude Detemination Based on Star Sensor/Gyro Integrated System[D], Harbin Institute of Technology, China, 2011

[5] Jun Ding, Research on Attitude Algorithm Based on Micro Inertial Sensors[D], Shanghai Jiao Tong University, China, 2013

[6] Rui Ma, Research on the Error Analysis and Compensation Technology Based on Micro Inertial Navigation System[D], Tsinghua University, China, 2010 